\title{
Detection and recognition of auditory patterns
}

\author{
DANIEL L. WEBER \\ Wright State University, Dayton, Ohio
}

\begin{abstract}
The detection/recognition theorem (Starr, Metz, Lusted, \& Goodenough, 1975) provides a signal detection theory prediction of an observer's ability to identify one of a set of possible signals on the basis of the observer's ability to detect whether any signal is presented. The present findings show that the theorem can be applied to complex auditory stimuli in a fashion that is not merely a trivial extension of the results obtained with simple auditory stimuli.
\end{abstract}

Starr and Metz (Starr, Metz, Lusted, \& Goodenough, 1975) developed the detection/recognition theorem to evaluate the performance of radiographic technicians faced with the task of identifying tumors in $\mathrm{X}$ rays. This task requires answers to two questions: (1) Is there a tumor present in the $X$ ray? and (2) Where is the tumor in the $X$ ray? The first question represents a simple detection task; the second question requires the identification of one signal (in this case, spatial location for the tumor) from a set of possible signals. Starr et al. (1975; Starr, Metz, \& Lusted, 1977) demonstrated that the theorem accurately predicted performance when the identification task required subjects to indicate which quadrant of the $X$ ray contained a simulated tumor. Extending the theorem to more complex visual stimuli, Swets, Green, Getty, and Swets (1978) reported that the theorem accurately predicted performance when the possible signals were visual patterns composed of sets of vertical lines drawn in different positions across a CRT screen.

\section{THE DETECTION/RECOGNITION THEOREM}

The detection/recognition theorem predicts the probability that an observer can correctly identify which one of $m$ possible signals was presented on the basis of the observer's ability to detect whether any signal was presented. The probability of a correct identification varies with the observer's detection criterion, which also determines the observer's position on the detection ROC curve. For each point $\left(F A R_{i}, H R_{i}\right)$ on the detection ROC curve, the theorem predicts the probability of correct identification, $P(c)$, to be

$$
P(c)=H R_{i}-\frac{m-1}{m} \int_{0}^{F A R_{i}} \frac{1-H R}{1-F A R} d F A R,
$$

\footnotetext{
I would like to thank Teresa Mayfield and Bob McKinley, who aided in collection and analysis of portions of these data. David Ansley provided valuable assistance in this research and reported part of these data at a meeting of the Acoustical Society of America (Ansley \& Weber, 1987). Larry Kurdek, Tom Moore, and an anonymous reviewer provided helpful comments during the preparation of this manuscript. This research was supported by AFOSR through AAMRL/BB at Wright-Patterson AFB. Correspondence about this paper may be addressed to the author at the Department of Psychology, Wright State University, Dayton, OH 45435.
}

where $F A R$ and $H R$ are, respectively, false-alarm-rate and hit-rate pairs on the detection ROC curve, and $F A R_{i}$ and $H R_{i}$ are, respectively, the false-alarm and hit rates determined by the selection of a particular detection criterion. Given that one has an ROC curve for the detection of any one of $m$ possible signals (illustrated in Figure 1a), the prediction of the theorem is obtained by finding the area under the $(1-H R) /(1-F A R)$ curve over the interval 0 to $F A R_{i}$ (illustrated in Figure 1b) and subtracting the appropriate fraction of this area from $H R_{i}$.

This form of the theorem derives from the assumptions that each of the $m$ possible signals may be considered to be detected by independent processes and that all the possible signals are equally detectable (derivations of the theorem can be found in Green, Weber, \& Duncan, 1977, and Starr et al., 1975). The independence assumption assumes that the possible signals are sufficiently separated along one or more dimensions (e.g., time, space, frequency), so that there is no influence of one possible signal upon the analysis of any other possible signal. This permits the detection and identification tasks to be discussed in terms of the performance of $m$ detectors, one for each of the $m$ signals. The equal detectability assumption is then expressed as the existence of equal false-alarm and hit rates (equal $d^{\prime}$ ) in each of the $m$ detectors.

The peripheral auditory system is frequently modeled as a set of band-pass filters. In such a model, it is easy to picture the independence assumption for the detection and recognition of simple auditory signals (sinusoids) as the requirement that the $m$ possible signals have frequencies that are well separated in terms of filter bandwidths (often related to critical bandwidths). Under these conditions, the events within filters with no common frequency components may be taken to be independent, and the identification task reduces to the identification of which filter contained the signal, given that any did. In signal detection terms, the detection task may be represented as the determination of whether the largest of the $m$ observed likelihood ratios (from the $m$ detectors for the $m$ possible signals) on each trial exceeds the detection criterion, and the identification task is the determination of which detector gave rise to the largest likelihood ratio. Green (Green et al., 1977) explored the application of such a representation of the detection/recognition theorem to sim- 


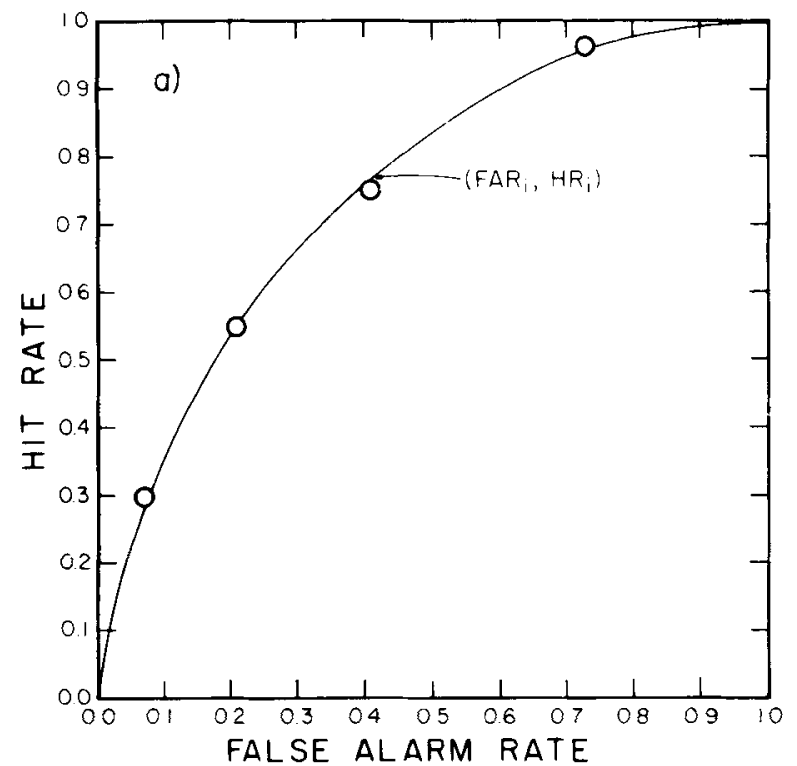

sinusoids that differ only in frequency, complex auditory stimuli of the type studied extensively by Watson (e.g., Watson, Kelly, \& Wroton, 1976; Watson et al., 1975). Unlike the case with sinusoids, the identification of auditory patterns generally cannot be modeled in terms of differences in their representations in the peripheral auditory system. Thus, the attempt to predict identification performance from detection performance for these auditory patterns might be considered to be an attempt to predict central (cognitive) performance from peripheral (sensory) response. The results show that this is possible in some cases.

\section{METHOD}

Four tonal patterns comprised the set of possible signals (Figure 2). Each pattern consisted of seven 100-msec sinusoids; the order of the frequencies distinguished the patterns. Two of the patterns were a descending glide (frequency decreased monotonically for the seven successive sinusoidal components, Pattern 2 in

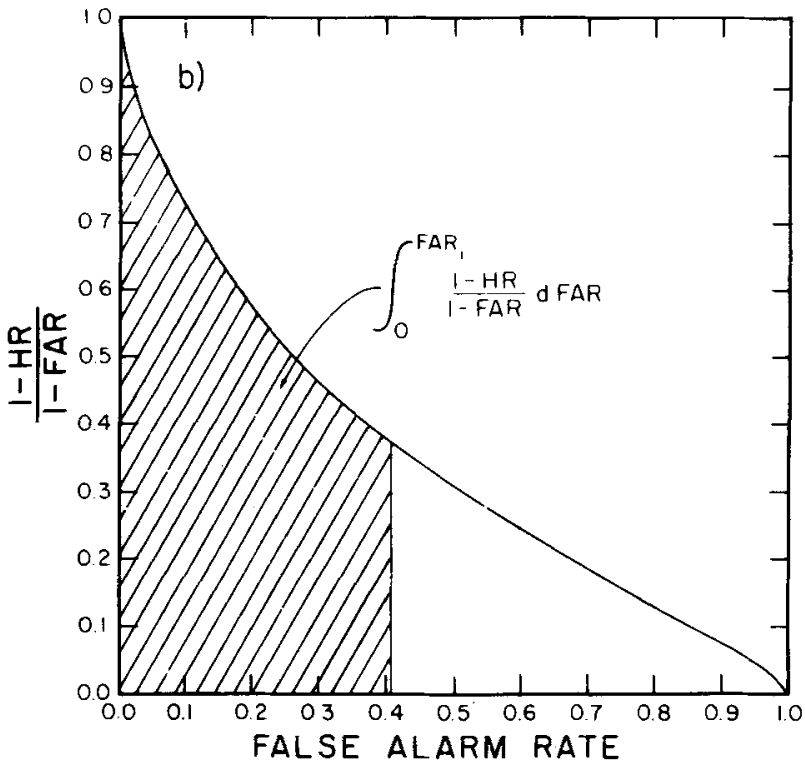

Figure 1. Prediction of probability of correct identification. The least squares fit $R O C$ curve in panel $a$ is used to generate the points that constitute the $(1-H R) /(1-F A R)$ curve in panel $b$. Each datum (circles in panel a) in the detection task arises from a different detection criterion. For each detection criterion, and thus $F A R_{i}$ for the ith datum, the detection/recognition theorem predicts the probability of a correct identification as the $H R_{i}$ value on the $R O C$ curve minus a fraction of the area under the $(1-H R) /(1-F A R)$ curve over the interval from 0 to $F A R_{i}$.

ple auditory signals and suggested the extension to complex auditory stimuli as a next step in the evaluation of the generality of the theorem.

As argued by Watson (e.g., Watson, Wroton, Kelly, \& Benbassat, 1975), a reasonable first step from single sinusoids toward more complex stimuli is to create sequences of sinusoids that vary on a single dimension. The experiment reported in this paper assessed whether the detection/recognition theorem can accurately predict performance for tonal patterns created from sequences of

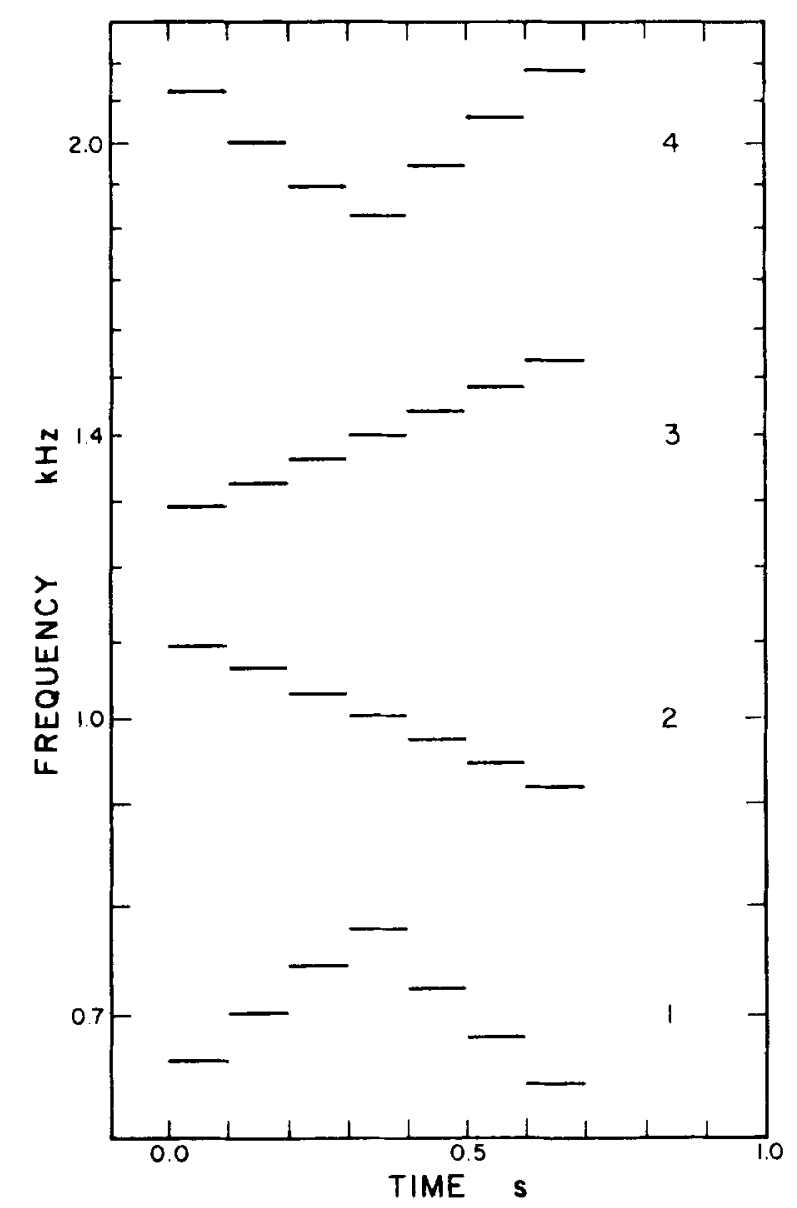

Figure 2. Tonal patterns created from sequences of sinusoids. For a set of seven frequencies used to create a pattern, the order of components was: Pattern 1, ascending glide followed by descending glide, 2, 4, 6, 7, 5, 3, 1; Pattern 2, descending glide, 7, 6, 5, 4, 3, 2, 1; Pattern 3, ascending glide, 1, 2, 3, 4, 5, 6, 7; Pattern 4, descending then ascending glide, $6,4,2,1,3,5,7$. These four patterns were used in each of nine conditions; frequencies for Condition $4 \mathrm{G}$ are shown. 
Figure 2) and an ascending glide (frequency increased for successive sinusoids, Pattern 3 ). The other two patterns contained two successive glides. One (Pattern 1) had an ascending glide followed by a descending glide and was created by presenting the evennumbered components in ascending order, then the odd-numbered components in descending order (given components frequencies 1 to 7 , the order was $2,4,6,7,5,3,1$ ). The other (Pattern 4) had a descending glide followed by an ascending glide (descending order on even-numbered components, then ascending on odd). These four patterns, always labeled "' 1 ," “'2," '“3," and ' 4 ," were used in all nine experimental conditions. A diotic, broadband (318-7800 Hz), white noise $\left(\mathrm{N}_{0}=20 \mathrm{~dB}\right.$ SPL) was continuously present during all testing. All signals were presented to the listener's left ear.

This experiment examined the accuracy of the predictions of the detection/recognition theorem as a function of the degree of frequency separation between the four tonal patterns. On the one extreme, the same seven sinusoids were used to create all four patterns. (Component frequencies for all patterns are shown in Figure 3. Condition 7A represents the case in which seven frequencies were common to all patterns.) On the other extreme, sinusoids within a pattern all fell within a half-octave interval, and there was a separation of a half octave between the components of successive patterns (Condition 4G in Figure 3). Between the extremes were conditions with smaller frequency gaps between patterns ( $G$ conditions), conditions with some number of frequencies shared by successive patterns but with no components common to all patterns ( $\mathrm{S}$ conditions), and conditions with some frequencies common to all patterns (A conditions).

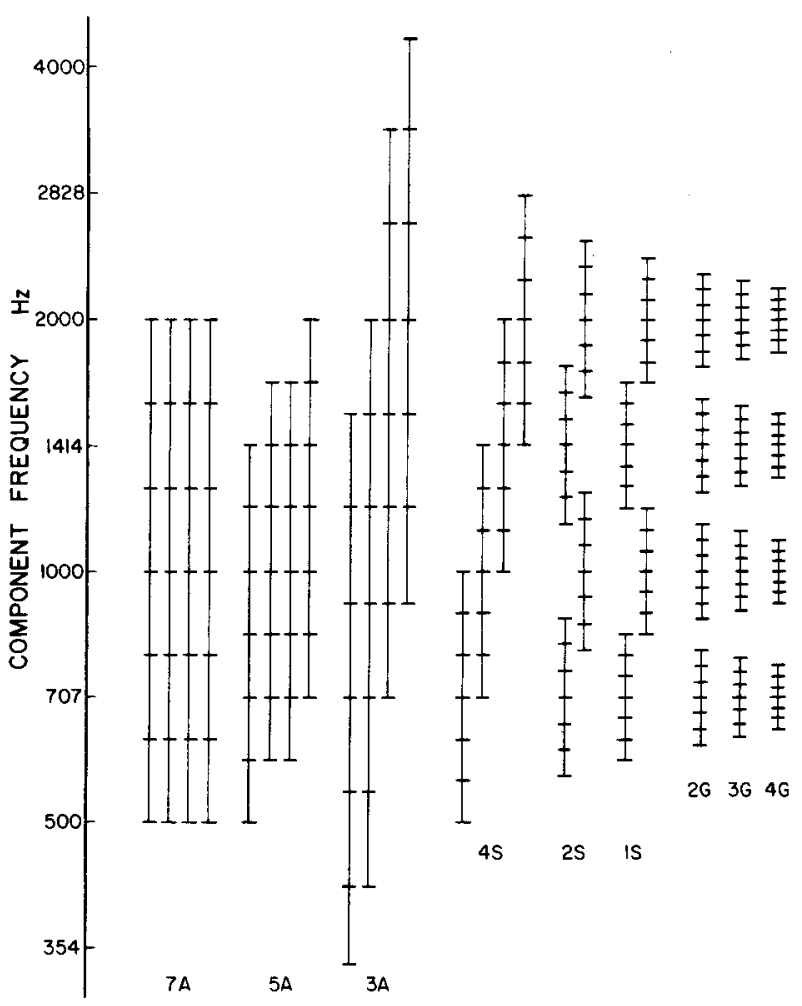

Figure 3. Signal frequencies used in nine stimulus conditions. Pattern numbers were: 1 , ascending then descending glide; 2 , ascending glide; 3 , descending glide; 4 , descending then ascending glide. Patterns with lower numbers were created from sets with lower frequencies.
When the components of different patterns lie within distinct frequency regions, pattern type is redundant with frequency region, and the identification task might be able to be performed without regard to pattern information per se. The necessary control condition, performance for four sinusoids $(707,1000,1414$, and $2000 \mathrm{~Hz}$, the center frequencies of the patterns in the $G$ and $S$ conditions and nearly those in Condition $3 \mathrm{~A}$ ), was also examined for comparison with the pattern data.

\section{The Detection/Recognition Task}

Each block of 80 trials began with four presentations of each of the four possible signals. During the block of trials, the probability of the presentation of some signal on a given trial was 0.5 , and each of the four signals was presented equally often (10 times in 80 trials).

On each trial, a $200-\mathrm{msec}$ warning and a $200-\mathrm{msec}$ pause preceded the 700 -msec observation interval. Separate lights indicated the warning, observation, and two response intervals. The first response was a rating on a scale of 1 to 5 of the listener's confidence that a signal had been presented. The second response indicated which signal was most likely to have given rise to the observation. The listeners were given unlimited time to respond. After all listeners had responded, feedback was provided by lighting ( $250 \mathrm{msec}$ ) an LED on the response button for the correct identification (for signal trials) or flashing the four control lights (for noise trials).

Green (Green et al., 1977) has shown that this two-response procedure permits efficient evaluation of the detection/recognition theorem. The rating-scale judgment provides a relatively quick way to obtain an estimate of the detection ROC curve (four points may be estimated from the five category data), and the identification data arise from the same observations as do the detection data.

\section{Data Analysis}

Each listener generated four points on a detection ROC curve in each of the nine experimental conditions and in the control condition. (As an example, data for D.D. in Condition 4S are shown in Figure 1a.) The data for the detection ROC curve were transposed into corresponding $z$ scores, so that the ROC curve could be represented as a straight line (Green \& Swets, 1966/1974). A least squares fit to the normal deviate values was used to produce a discrete approximation of points on the curve $(1-H R) /(1-F A R)$ as a function of false-alarm rate (Figure 1 b) every $1 / 100$ of a unit. As $F A R$ increased from 0 to 1 in increments of 0.01 , each value of $F A R$ was converted into a $z$ score, which was used as the value of the independent variable in the ROC curve regression line. The value of $H R$ corresponding to the $z$ score predicted from the regression equation was then used to represent the detection ROC curve (seen as a smooth curve in Figure 1a) and to calculate a point on the $(1-H R) /(1-F A R)$ curve (the smooth curve in Figure $1 \mathrm{~b})$. The area under this curve was estimated by 0.01 , the bin width for $F A R$, multiplied by the average of the value estimated for $(1-H R) /$ $(1-F A R)$ and the value at the preceding point. It was taken as a given that when $F A R$ equals $0, H R$ equals 0 and the ratio $(1-H R) /(1-F A R)$ equals 1 .

For each detection criterion adopted by the listener and for a criterion likelihood ratio of zero (that is, for each $F A R_{i}$ equal to the value of $F A R$ for a datum and for $F A R_{i}$ equal to one), an estimate of the probability of a correct identification was obtained by subtracting three fourths $[(4-1) / 4]$ of the area under the $(1-H R) /(1-F A R)$ curve over the interval $\left(0, F A R_{i}\right)$ from the $H R_{i}$ generated from the regression equation.

Because the absolute value of these predictions starts at zero, there is a ceiling effect in the magnitude of simple differences between the predicted and obtained probabilities of a correct identification for small values of $F A R$. To avoid this difficulty, the accuracy of prediction reported in this paper compares the obtained performance 
with the predicted performance as percentage error calculated as obtained minus predicted divided by the predicted probability. This measure is obtained for each datum on the ROC curve (including the point 1,1 ). Although the simple difference increases systematically with $F A R$, percent error is essentially constant, and performance for the condition is reported as the mean of the five estimates obtained for each ROC curve.

Each detection ROC curve is based on at least 1,120 observations obtained over three sessions. Prior to these observations, the listeners received three to five 2-h sessions of practice with the stimuli for the condition to be tested. The first session with the stimuli in a new condition started with practice in the identification task, with the stimuli presented 10 to $15 \mathrm{~dB}$ above threshold. After all listeners achieved $100 \%$ correct identifications, the signal levels were gradually reduced to the level identified in the first condition to yield a detection ROC curve with a $d^{\prime}$ between 1 and 2. Typically, two or three sessions were required to get the listeners accustomed to this level. When the listeners had completed at least two practice sessions at the selected level, data collection for that condition began and continued until a minimum of 1,120 observations had been performed. After all listeners in a group reached this criterion, a new condition was started. The 10 conditions were tested in different random orders for the two groups of listeners. This amount of practice should have been more than sufficient for the listeners to learn these simple patterns (Leek \& Watson, 1988). There was no evidence of any practice effect within or across conditions.

\section{Listeners}

All listeners were college-age individuals with clinically young, normal hearing at frequencies in the range of 250 to $4000 \mathrm{~Hz}$. All were paid for their participation. Each listener was tested for at least $30 \mathrm{~h}(20 \mathrm{~h}$ for practice and measurement of thresholds for individual components and $10 \mathrm{~h}$ of practice on the detection/recognition task) before collection of any data reported here. Six female listeners, tested in groups of three, participated in the study. In one group, partners were D.D., T.M., and P.P., and in the other, A.P., C.M., and D.V. Fifteen additional listeners ( 7 female, 8 male) were tested in the four-tone condition.

\section{Stimulus Generation}

The output of a Grason Stadtler (Model 445C) white-noise generator was band-pass filtered (two Ithaco Model 4302 filters in series; the obtained 3-dB bandwidth was 318 to $7800 \mathrm{~Hz}$ ) and attenuated for diotic presentation at a noise spectrum level of $20 \mathrm{~dB}$ SPL. This background noise was present continuously throughout each session.

The sinusoidal signals were generated digitally at a presentation rate of 20,000 points per second. All sinusoids began and ended at a positive-going zero crossing. Successive components in a pat- tern were presented without any temporal separation; the positivegoing waveform for one component ended at the zero crossing, and the onset of the next waveform began with the same point. No ramps were applied to the components.

The PDP-11/73 computer that controlled all aspects of event timing and response collection output digital values through a Boys Town (BTNI Model 1000) DMA control board and DAC. The DAC output was low-pass filtered at $3150 \mathrm{~Hz}$ (Ithaco Model 4302 filter) then split into four separate paths through a homebuilt emitterfollower circuit. Each channel was attenuated to an appropriate level for each headphone/ear combination before being added to the background noise. Separate channels of four Crown D-75 amplifiers transmitted the signal and noise to the listeners seated in separate sound-isolating chambers (either IAC Model 1200 or 400). Attenuators in the chambers reduced transmission noise before the signals were presented through matched pairs of TDH-39 headphones in standard 001 cushions.

\section{Threshold Estimation}

The derivation of the detection/recognition theorem assumes that all possible signals are equally detectable. Most (75\%) of the components were presented within $\pm 1 \mathrm{~dB}$ of equal sensation level; only the $500-\mathrm{Hz}$ component ever exceeded $2 \mathrm{~dB}(-2.6 \mathrm{~dB}$ for T.M. and $2.7 \mathrm{~dB}$ for C.M.). This was achieved by measuring thresholds for each individual component for each listener and adjusting component levels to compensate for the group performance. Threshold values, compensation factors, and stimulus levels used in the presentation of the patterns are given in Table 1.

Thresholds were measured in an adaptive, two-interval, forcedchoice procedure. Each trial began with a 150 -msec warning and a 150 -msec pause, followed by the two 100 -msec observation intervals. The observation intervals were separated by $300 \mathrm{~ms}$. Following the second observation, the listeners were able to respond by pressing a button corresponding to the interval that they believed to contain the signal. The warning, observation, and response intervals were indicated by separate LEDs. After all listeners responded, feedback was provided by an LED that flashed $(150 \mathrm{msec})$ on the appropriate response button. After a 150 -msec pause, the next trial began.

Signal level varied across trials depending upon the listener's performance. The initial level was set 15 to $20 \mathrm{~dB}$ above the expected threshold. Thereafter, every two correct responses at a given level resulted in a reduction in signal level, and each incorrect response resulted in an increase. The initial change was an 8-dB step. After two reversals in the direction of change (e.g., going from a series of correct responses to an incorrect response), the change was reduced to a $2-\mathrm{dB}$ step. All subsequent stimulus levels for which a reversal occurred were averaged to produce a threshold estimate for that sequence of $\mathbf{5 0}$ trials.

Table 1

Masked Threshold for 100-msec Components

\begin{tabular}{cccccccc}
\hline \multirow{2}{*}{$\begin{array}{c}\text { Signal } \\
\text { Frequency }\end{array}$} & D.D. & T.M. & P.P. & D.V. & A.P. & C.M. & Compensation \\
\hline 500 & 35.6 & 39.9 & 35.5 & 34.8 & 35.5 & 29.3 & 4.9 \\
700 & 39.5 & 39.4 & 37.3 & 37.6 & 34.8 & 32.9 & 3.2 \\
850 & 39.3 & 39.3 & 40.0 & 38.7 & 37.6 & 34.8 & 2.5 \\
1000 & 40.0 & 40.0 & 39.9 & 37.1 & 37.9 & 36.9 & 2.0 \\
1200 & 41.2 & 41.1 & 40.6 & 40.2 & 38.8 & 34.3 & 1.0 \\
1400 & 42.2 & 42.5 & 40.0 & 38.9 & 38.6 & 35.6 & 0.4 \\
1650 & 42.2 & 42.7 & 40.9 & 39.3 & 39.4 & 35.3 & 0 \\
2000 & 41.8 & 40.0 & 40.7 & 40.8 & 39.9 & 35.2 & 1.7 \\
\multicolumn{7}{c}{ Level of 1000-Hz Component in dB SPL Across Patterns } & \\
\hline
\end{tabular}

Note-Signal threshold is given in dB SPL; signal frequency is expressed in $\mathrm{Hz}$; and compensation is in $\mathrm{dB}$. 
Two 50-trial blocks were presented consecutively for each con dition. Blocks of trials yielding fewer than 10 reversals or standard deviations greater than $5 \mathrm{~dB}$ were not considered to yield valid threshold estimates and were not included in the final threshold estimate, which was the average of at least six estimates obtained on three different days. If the standard deviation for the six estimates was greater than $5 \mathrm{~dB}$, four additional estimates were obtained. In the data in Table $1,88 \%$ of the estimates have standard deviations under $3 \mathrm{~dB}$, and all have standard deviations under $4 \mathrm{~dB}$.

\section{RESULTS AND DISCUSSION}

The accuracy of the predictions of the detection/recognition theorem is represented in Figure 4, with one panel for each of the six listeners. Within each panel, the average percent error for the five points on the detection ROC curve for each of the nine pattern conditions is plotted as a circle with an error bar representing one standard

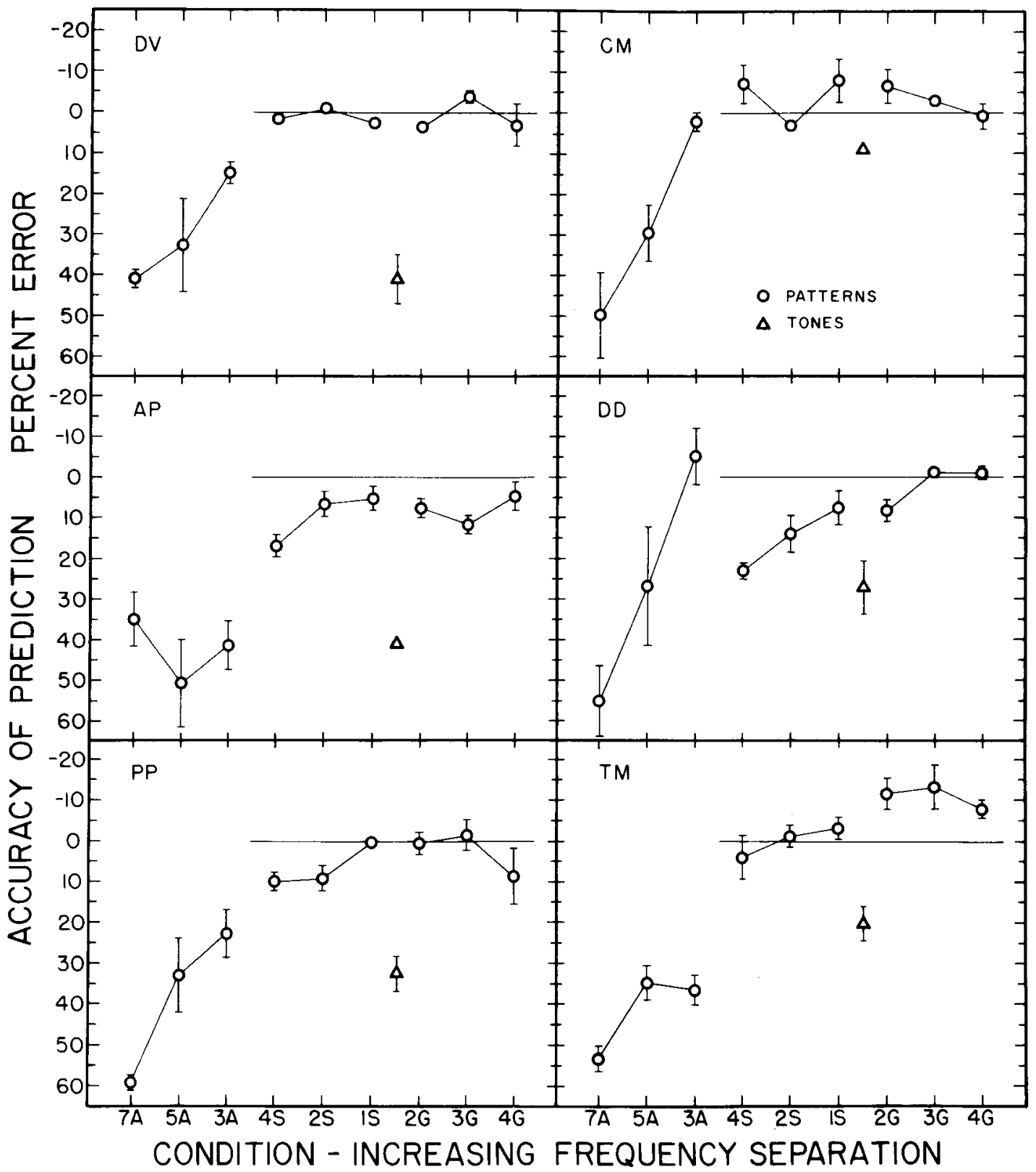

Figure 4. Accuracy of prediction of detection/recognition theorem in nine conditions for each of 6 listeners. Data (circles for auditory patterns, triangles for four tones) below zero (horizontal line) represent performance below predicted level. Error bars omitted when smaller than symbol. Lines connect data from similar conditions. 
deviation. The horizontal line at zero percent error provides a convenient reference; data below this line represent performance by the listener that fell below the predicted value. Performance in the four-tone control condition is represented by a triangle.

Although there was ample variability among listeners, several trends were apparent. When performance of the identification task depended entirely on resolution of the sequence of frequencies themselves (Condition 7A, in which the four patterns were composed of the same seven frequencies presented in different orders), the listeners performed at a much lower level than that predicted by the detection/recognition theorem. However, when the frequencies within a pattern were confined to discrete and separate frequency regions (the $G$ conditions), the same listeners matched the performance predicted by the theorem.

This good performance might have extended to conditions in which there was substantial overlap among the frequencies of successive patterns but no frequencies common to all patterns (the $S$ conditions). This certainly is evident in the data of D.V. and C.M. (Figure 4), and consistent performance in the $G$ and $S$ conditions was seen for P.P. and A.P. (Figure 4). Listener T.M. (Figure 4) clearly showed performance at the predicted level for the condition of greatest overlap between successive components, but her better-than-predicted performance in the $G$ conditions prevents a straightforward interpretation of the data. D.D. (Figure 4) also offered data that are difficult to interpret, because her performance in the Condition 3A matched the prediction of the theorem, yet her performance on the presumably easier $S$ conditions fell far below that predicted. Nonetheless, the general result is that performance was good when there was frequency separation between patterns and remained at a fairly constant level until there was substantial overlap between the frequencies used in the different patterns.

Such a result is consistent with a peripheral representation of the identification task. Even when two and four of the seven frequencies in a pattern were shared with successive patterns (Conditions $2 S$ and $4 S$ ), the center frequencies of the patterns remained a half octave apart, as in all the $\mathbf{G}$ conditions. Any analysis of central tendency of the frequencies in a pattern, totally ignoring their order, would lead to constant identification performance across these conditions. In this context, pattern identification could be modeled by identification of activity in the filter representing the center frequency of the pattern.

This interpretation is consistent with the single previous test of the detection/recognition theorem with auditory stimuli (Green et al., 1977). However, it does not provide a satisfactory explanation for the data in this experiment for two reasons. First, the explanation fails to account for the poor performance on Condition 3A, which had the same center frequencies as the $S$ and $G$ conditions. (That D.D. showed such good performance on Condition $3 \mathrm{~A}$ is not support for this argument, since D.D. did not show the consistent performance across conditions that this interpretation would predict.) Second, and more importantly, the good performance in the gap conditions cannot be attributed solely to an "easy" peripheral representation of the stimuli for the identification task, because the four-tone control condition, which clearly depended only on such a representation, yielded poor performance. (This is true even for the data for C.M. An ANOVA shows the values for the $3 \mathrm{~A}, \mathrm{~S}, \mathrm{G}$, and fourtone conditions for C.M. to be significantly different $p<.01$.)

The poor performance on the four-tone condition was unexpected, because good performance is easily interpreted in terms of the filter model of peripheral frequency analysis and because performance matching that predicted by the detection/recognition theorem had been reported (Green et al., 1977). Because the interpretation of data in Figure 3 depends so heavily on comparison to performance in the four-tone condition, additional listeners were tested to see whether the data in this experiment could be considered to be representative. The results for the 4 listeners in the Green et al. (1977) experiment, the 6 listeners in this experiment, and 15 additional listeners are ranked by goodness of performance in Table 2.

Performance on the identification task does not appear to be related to any aspect of the detection task; all of the listeners operated within a small range of $d^{\prime}$ values and distributed their criteria in a comparable fashion. The listeners tested by Green et al. were superior to any others

Table 2

Detection and Rank Ordering of Identification Performance with Four Sinusoids

\begin{tabular}{|c|c|c|c|c|}
\hline \multirow[b]{2}{*}{$\underline{\text { Listener }}$} & \multirow[b]{2}{*}{$d_{e}^{\prime}$} & \multicolumn{2}{|c|}{ Criteria } & \multirow{2}{*}{$\begin{array}{l}\text { Identification } \\
\text { Percent Error }\end{array}$} \\
\hline & & Maximum & Minimum & \\
\hline G.R.* & 0.92 & 4.76 & 0.55 & 0.3 \\
\hline L.T.* & 1.64 & 5.83 & 0.47 & 2.2 \\
\hline K.L.* & 0.99 & 9.25 & 0.54 & 4.5 \\
\hline J.U.* & 1.06 & 4.41 & 0.62 & 6.4 \\
\hline C.M.† & 0.91 & 5.36 & 0.39 & 9.6 \\
\hline C.H. & 1.25 & 2.66 & 0.76 & 11.0 \\
\hline D.Z. & 1.17 & 4.20 & 0.86 & 14.9 \\
\hline T.M. $\dagger$ & 1.12 & 3.73 & 0.49 & 20.1 \\
\hline K.H. $\ddagger$ & 1.60 & 3.85 & 0.41 & 20.4 \\
\hline P.T. & 0.96 & 4.95 & 0.67 & 21.2 \\
\hline B.P. $\ddagger$ & 1.24 & 2.82 & 0.91 & 21.5 \\
\hline R.K. & 1.00 & 5.84 & 0.49 & 21.8 \\
\hline D.B. & 1.16 & 4.33 & 0.44 & 22.0 \\
\hline B.S. & 1.03 & 1.95 & 0.68 & 22.3 \\
\hline T.R. & 1.12 & 359 & 0.66 & 23.0 \\
\hline K.S. & 1.22 & 5.17 & 0.84 & 25.3 \\
\hline D.W. & 0.73 & 4.35 & 0.46 & 26.0 \\
\hline L.S. & 0.80 & 1.63 & 0.39 & 27.1 \\
\hline D.A. & 1.06 & 3.91 & 0.49 & 27.7 \\
\hline D.D.† & 0.76 & 2.04 & 0.48 & 27.9 \\
\hline P.P.† & 0.83 & 3.84 & 0.80 & 28.6 \\
\hline E.G. $\ddagger$ & 1.26 & 1.80 & 0.70 & 31.2 \\
\hline B.K. & 0.99 & 3.31 & 0.86 & 33.2 \\
\hline D.V.† & 0.98 & 13.70 & 0.72 & 40.7 \\
\hline A.P.† & 1.16 & 21.37 & 0.70 & 42.3 \\
\hline
\end{tabular}

*Data from the Green, Weber, and Duncan (1977) study. †Data from the present study. $\ddagger$ Data collected in the Psychoacoustic Laboratory, AAMRL/BBA, Wright-Patterson AFB. 
tested. The listeners in the present experiment ranged from the best (C.M.) to the worst (A.P.) of the remaining listeners. The data for C.M. appear to approach consistency with a description of the identification task based upon a peripheral representation of the stimuli (although an ANOVA shows the data do not support this interpretation). Listener C.M.'s data are closest to the performance of the listeners tested by Green et al., and it may be that those listeners would produce data in the pattern conditions that would be consistent with a peripheral representation of the identification task. However, these listeners were much better than the typical listener in the four-tone condition, and the data for the majority of the listeners in the present study seem likely to be representative of the majority of listeners.

\section{Failure of the Theorem}

The detection/recognition theorem failed to make good predictions in cases in which the performance of the listeners fell below the predicted level of performance. This mismatch between prediction and performance is reasonably attributed to an application of the theorem in conditions that violate assumptions made in the derivation of the theorem. The experimental design sought to achieve obedience to the equal detectability assumption by attempting to present all components in all patterns at the same sensation level. Measurement of $d^{\prime}$ values for detection ROC curves showed good consistency across conditions (Table 3). Determination of an appropriate false-alarm rate for different patterns within a condition is problematical. However, hit rates for the different patterns within a condition can provide an estimate of consistency. It appears that these values are not as close to constant as one would like (Table 3). The patterns in Condition $7 \mathrm{~A}$ had to be equally detectable, because the same components were used in all patterns and the wide frequency separation of components presented near threshold level precluded any interaction among them. Using this condition as an indication of variability in hit rates for equally detectable signals shows that detectability was roughly constant for most conditions. Given that the equal detectability assumption was satisfied, failure of the theorem can be attributed to violation of the independence assumption.

The independence assumption in the derivation of the theorem requires statistical independence among the detectors that process the $m$ possible signals. It is not at all clear how independence should be predicted when the

Table 3

Hit Rates per Pattern and $d$ :

\begin{tabular}{|c|c|c|c|c|c|c|c|c|c|c|c|}
\hline \multirow[b]{2}{*}{ Listener } & \multirow[b]{2}{*}{ Pattern } & \multicolumn{10}{|c|}{ Condition } \\
\hline & & 7A & $5 \mathrm{~A}$ & $3 A$ & $4 S$ & $2 S$ & $1 S$ & $2 G$ & $3 G$ & $4 G$ & Four-Tone \\
\hline D.D. & $\begin{array}{c}1 \\
2 \\
3 \\
4 \\
d !\end{array}$ & $\begin{array}{l}.2 \\
.223 \\
.285 \\
.169 \\
.44\end{array}$ & $\begin{array}{l}.277 \\
.369 \\
.238 \\
.323 \\
.27\end{array}$ & $\begin{array}{l}.993 \\
.954 \\
.132 \\
.193 \\
.73\end{array}$ & $\begin{array}{c}.584 \\
.484 \\
.592 \\
.539 \\
1.02\end{array}$ & $\begin{array}{c}.415 \\
.530 \\
.770 \\
.493 \\
1.03\end{array}$ & $\begin{array}{l}.527 \\
.656 \\
.934 \\
.553 \\
1.14\end{array}$ & $\begin{array}{c}.908 \\
.854 \\
.976 \\
.901 \\
1.20\end{array}$ & $\begin{array}{c}.976 \\
.823 \\
.723 \\
.288 \\
1.38\end{array}$ & $\begin{array}{l}.82 \\
.715 \\
.515 \\
.19 \\
1.02\end{array}$ & $\begin{array}{l}.733 \\
.420 \\
.400 \\
.326 \\
.76\end{array}$ \\
\hline T.M. & $\begin{array}{c}1 \\
2 \\
3 \\
4 \\
d^{\prime}\end{array}$ & $\begin{array}{l}.336 \\
.326 \\
.26 \\
.362 \\
.58\end{array}$ & $\begin{array}{l}.208 \\
.323 \\
.346 \\
.346 \\
.20\end{array}$ & $\begin{array}{l}.562 \\
.631 \\
.354 \\
.354 \\
.96\end{array}$ & $\begin{array}{l}.446 \\
.439 \\
.492 \\
.500 \\
1.01\end{array}$ & $\begin{array}{l}.292 \\
.477 \\
.623 \\
\\
.92\end{array}$ & $\begin{array}{l}.447 \\
.56 \\
.647 \\
.64 \\
1.13\end{array}$ & $\begin{array}{c}.885 \\
.992 \\
.954 \\
.924 \\
1.02\end{array}$ & $\begin{array}{c}.847 \\
.624 \\
.465 \\
.447 \\
1.03\end{array}$ & $\begin{array}{l}.77 \\
.675 \\
.51 \\
.44 \\
1.03\end{array}$ & $\begin{array}{l}.546 \\
.484 \\
.354 \\
.515 \\
1.12\end{array}$ \\
\hline P.P. & $\begin{array}{r}1 \\
2 \\
3 \\
4 \\
d^{\prime}\end{array}$ & $\begin{array}{l}.615 \\
.723 \\
.523 \\
.554 \\
.88\end{array}$ & $\begin{array}{l}.562 \\
.321 \\
.446 \\
.434 \\
.44\end{array}$ & $\begin{array}{c}.915 \\
.938 \\
.527 \\
.415 \\
1.29\end{array}$ & $\begin{array}{c}.772 \\
.700 \\
.793 \\
.671 \\
1.25\end{array}$ & $\begin{array}{c}.484 \\
.707 \\
.915 \\
.769 \\
1.17\end{array}$ & $\begin{array}{l}.46 \\
.693 \\
.933 \\
.794 \\
1.11\end{array}$ & $\begin{array}{r}.824 \\
.854 \\
.946 \\
.962 \\
1.13\end{array}$ & $\begin{array}{c}.929 \\
.834 \\
.729 \\
.435 \\
1.12\end{array}$ & $\begin{array}{l}.794 \\
.719 \\
.699 \\
.358 \\
1.09\end{array}$ & $\begin{array}{l}.684 \\
.616 \\
.383 \\
.459 \\
.83\end{array}$ \\
\hline D.V. & $\begin{array}{c}1 \\
2 \\
3 \\
4 \\
d^{\prime}\end{array}$ & $\begin{array}{l}.550 \\
.567 \\
.591 \\
.496 \\
.77\end{array}$ & $\begin{array}{l}.443 \\
.350 \\
.307 \\
.379 \\
.35\end{array}$ & $\begin{array}{l}.958 \\
.842 \\
.617 \\
.425 \\
.81\end{array}$ & $\begin{array}{l}.82 \\
.86 \\
.934 \\
.826 \\
1.34\end{array}$ & $\begin{array}{l}.531 \\
.812 \\
.95 \\
.9 \\
1.19\end{array}$ & $\begin{array}{c}.72 \\
.873 \\
.973 \\
.94 \\
1.27\end{array}$ & $\begin{array}{l}.781 \\
.787 \\
.969 \\
.957 \\
1.32\end{array}$ & $\begin{array}{l}.590 \\
.575 \\
.957 \\
.986 \\
1.09\end{array}$ & $\begin{array}{l}.783 \\
.549 \\
.592 \\
.125 \\
.92\end{array}$ & $\begin{array}{l}.75 \\
.589 \\
.548 \\
.40 \\
.98\end{array}$ \\
\hline A.P. & $\begin{array}{c}1 \\
2 \\
3 \\
4 \\
d !\end{array}$ & $\begin{array}{l}.409 \\
.454 \\
.380 \\
.353 \\
.26\end{array}$ & $\begin{array}{l}.379 \\
.378 \\
.364 \\
.421 \\
.30\end{array}$ & $\begin{array}{l}.641 \\
.633 \\
.492 \\
.392 \\
.85\end{array}$ & $\begin{array}{l}.401 \\
.533 \\
.845 \\
.649 \\
.97\end{array}$ & $\begin{array}{l}.27 \\
.614 \\
.868 \\
.836 \\
.93\end{array}$ & $\begin{array}{l}.10 \\
.447 \\
.773 \\
.8 \\
.95\end{array}$ & $\begin{array}{l}.176 \\
.419 \\
.806 \\
.85 \\
.95\end{array}$ & $\begin{array}{l}.478 \\
.543 \\
.936 \\
.950 \\
.82\end{array}$ & $\begin{array}{l}.959 \\
.867 \\
.759 \\
.333 \\
.77\end{array}$ & $\begin{array}{c}.65 \\
.552 \\
.457 \\
.443 \\
1.16\end{array}$ \\
\hline C.M. & $\begin{array}{c}1 \\
2 \\
3 \\
4 \\
d !\end{array}$ & $\begin{array}{l}.290 \\
.233 \\
.250 \\
.216 \\
.27\end{array}$ & $\begin{array}{l}.393 \\
.479 \\
.30 \\
.364 \\
.30\end{array}$ & $\begin{array}{l}.892 \\
.825 \\
.408 \\
.400 \\
.43\end{array}$ & $\begin{array}{l}.713 \\
.633 \\
.696 \\
.8 \\
.83\end{array}$ & $\begin{array}{l}.612 \\
.712 \\
.894 \\
.831 \\
.85\end{array}$ & $\begin{array}{l}.463 \\
.56 \\
.686 \\
.84 \\
.94\end{array}$ & $\begin{array}{l}.481 \\
.575 \\
.775 \\
.919 \\
.93\end{array}$ & $\begin{array}{l}.532 \\
.511 \\
.870 \\
.907 \\
.82\end{array}$ & $\begin{array}{l}.941 \\
.666 \\
.741 \\
.459 \\
.83\end{array}$ & $\begin{array}{l}.777 \\
.622 \\
.478 \\
.583 \\
.91\end{array}$ \\
\hline
\end{tabular}

Note-Hit rates evaluated at second most strict criterion used by each listener. Overall performance represented by $d$ ! for ROC curve. 
stimuli are auditory patterns. One possibility is that features of a pattern (e.g., glide direction, frequency extent of glide, temporal extent of glide, frequency region of glide, number of glides, etc.) might be assigned values on different dimensions of an $n$ dimensional space representing auditory stimuli. Within such a framework, independence would have an operational definition in terms of the extent that the representation (and determination of a likelihood ratio for the occurrence) of one stimulus would interact with the representation (and analysis) of another stimulus.

Despite the lack of such a model, the accuracy of the detection/recognition theorem may still provide useful information about the representation of complex auditory stimuli (Green et al., 1977). Given that the degree of underperformance by listeners is related to the degree of nonindependence (correlation or similarity) in the representation of stimuli, the detection/recognition theorem provides a metric with which to assess distances between complex stimuli that are sufficiently close to interact. Applied to the present study, this approach indicates that the combination of the dimensions of frequency sequence (pattern in time) and frequency region in the stimuli in the $S$ and $G$ conditions produced stimuli sufficiently far apart to satisfy the independence assumption. Frequency sequence and frequency region by themselves did not do so, and frequency sequence (Condition 7A) produced less distinct representations than did frequency region (four-tone condition). With more data, one could attempt to scale these distances in terms of the degree of inaccuracy in the predictions.

To complete such a modeling attempt, additional variables must be considered. Two avenues of research currently under investigation are temporal patterns (at a given frequency region) and different types of frequency sequences. These additional data should provide the basic information necessary for a predictive model of the representation of complex stimuli based upon the accuracy of the detection/recognition theorem.

The detection/recognition theorem may have practical applications independent of such theoretical issues. Auditory warning signals are used to convey information about sources of danger in a variety of settings (such as a control room at a nuclear power plant). Although it remains to be tested, stimuli that lead to performance matching the predictions of the detection/recognition theorem may yield fewer identification errors (because of their presumed independence). Such stimuli would be preferred as warning signals.

\section{CONCLUSION}

The detection/recognition theorem accurately predicts the performance of humans listening to certain types of complex auditory stimuli. The results of the present study are not consistent with an explanation based on an exclusively peripheral analysis of auditory stimuli, but represent the prediction of some type of central analysis based on peripheral performance. The failure of the detection/ recognition theorem to predict performance accurately for other auditory patterns can provide a method to assess the separation of these stimuli in whatever space is used to represent complex sounds.

\section{REFERENCES}

ANSLEY, D. A., \& WEBER, D. L.(1987). Detection and recognition of complex stimuli. Journal of the Acoustical Society of America, 82, S93(A).

GREEN, D. M., \& Swets, J. A. (1974). Signal detection theory and psychophysics (rev. ed.). Melbourne, FL: Krieger. (Original work published 1966)

Green, D. M., Weber, D. L., \& Duncan, J. E. (1977). Detection and recognition of pure tones in noise. Journal of the Acoustical Society of America, 62, 948-954.

LEEK, M. R., \& WATSON, C. S. (1988). Auditory perceptual learning of tonal patterns. Perception \& Psychophysics, 43, 389-394.

Starr, S. J., Metz, C. E., \& Lusted, L. B. (1977). Comments on the generalization of receiver operating characteristics analysis to detection and localization tasks. Physics in Medicine \& Biology, 22, 376-381.

Starr, S. J., Metz, C. E., Lusted, L. B., \& Goodenough, D. J. (1975). Visual detection and localization of radiographic images. Radiology, 116, 533-538.

Swets, J. A., Green, D. M., GetTy, D. J., Swets, J. B. (1978). Signal detection and identification at successive stages of observation. Perception \& Psychophysics, 23, 275-289.

Watson, C. S., Kelly, W. J., \& Wroton, H. W. (1976). Factors in the discrimination of tonal patterns. II. Selector attention and learning under various levels of stimulus uncertainty. Joumal of the Acoustical Society of America, 60, 1176-1186.

Watson, C. S., Wroton, H. W., Kelly, W. J., a Benbassat, C. A. (1975). Factors in the discrimination of tonal patterns. I. Component frequency, temporal position, and silent intervals. Journal of the Acoustical Society of America, 57, 1175-1185.

(Manuscript received July 14, 1988; revision accepted for publication December 15, 1988.) 